\title{
Effect of Hydromagnetic Mixed Convection Double Lid-Driven Square Cavity with Inside Elliptic Heated Block
}

\author{
M. J. H. Munshi ${ }^{1 *}$, M. A. Alim ${ }^{2}$ \\ ${ }^{1}$ Department of Mathematics, Hamdard University Bangladesh (HUB), Hamdard Nagar, \\ Gazaria, Munshigonj- 1510, Bangladesh \\ ${ }^{2}$ Department of Mathematics, Bangladesh University of Engineering and Technology (BUET), \\ Dhaka- 1000, Bangladesh
}

Received 12 May 2016, accepted in final revised form 22 September 2016

\begin{abstract}
Mixed convection heat transfer in a two-dimensional the effect of hydromagnetic mixed convection in a double lid driven square cavity with inside elliptic heated block is studied numerically. The left wall of the square cavity and inside the elliptic block was kept at $T_{h}$ while the right wall of the square cavity at a cold temperature $T_{c}$ with $T_{h}>T_{c}$. The lid is assumed to be upper wall moving in left to right and lower wall right to left directions respectively. The magnetic field of strength $B$ is applied parallel to $x$-axis. Result is presented firstly for different Reynolds number ( $50 \leq R e \leq 150)$, Prandtl number $\operatorname{Pr}=0.733$ and presented for different Grashof number $\left(10^{3} \leq \mathrm{Gr} \leq 10^{6}\right)$. The numerical results studied the effect of Reynolds number, Grashof number and buoyancy ratio on the local values. It is found that direction of lid and different elliptic heated block are more effective on heat and mass transfer on fluid flow with increasing magnetic field for all studied parameters.
\end{abstract}

Keywords: Mixed convection; Square cavity; Elliptic obstacle; Lid-driven cavity and FEM.

(C) 2017 JSR Publications. ISSN: 2070-0237 (Print); 2070-0245 (Online). All rights reserved. doi: http://dx.doi.org/10.3329/jsr.v9i1.27702 J. Sci. Res. 9 (1), 1-11 (2017)

\section{Introduction}

Mixed convection in enclosures is encountered in many engineering systems such as cooling of electronic components, ventilation in building and fluid movement in solar energy collectors, astrophysics, geology, biology, and chemical processes, as well as in many engineering applications such as solar ponds, crystal manufacturing, and metal

Corresponding author: jahir.buet.bd@gmail.com 
solidifications processes. Also mixed convection involving the combined effect of forced and natural convection has been the focus of research due to its occurrence in numerous technological, engineering, and natural applications such as: cooling of electronic devices, lubrication technologies, drying technologies, food processing, float glass production, etc. [1-3]. Al-Amiri et al. [4] investigated numerically steady mixed convection in a square lid-driven cavity under the combined buoyancy effects of thermal and mass diffusion. The results demonstrate the range where high heat and mass transfer rates can be attained for a given Richardson number. Sharif [5] studied numerically laminar mixed convective heat transfer in two-dimensional shallow rectangular driven cavities of aspect ratio 10 . The top moving lid of the cavity is at a higher temperature than the bottom wall. The effects of inclination of the cavity on the flow and thermal fields are investigated. The stream line and isotherm plots and the variation of the local and average Nusselt numbers at the hot and cold walls are presented. Chen and Cheng [6] investigated numerically the periodic behavior of the mixed convective flow in a rectangular cavity with a vibrating lid. The periodic flow patterns and heat transfer characteristics found are discussed with attention being focused on the interaction between the frequency of the lid velocity vibration and the frequency of the natural periodic flow. Khanafer et al. [7] investigated numerically unsteady laminar mixed convection heat transfer in a lid driven cavity. Teamah et al. [8] analyzed the numerical simulation of double-diffusive mixed convective flow in rectangular enclosure with insulated moving lid. Saha et al. [9] have performed the numerical effect of internal heat generation or absorption on MHD (magnetohydrodynamics) mixed convection flow in a lid driven cavity. The significant reduction in the average Nusselt number were produced as the strength of the applied magnetic field was increased. In addition, heat generation predicated to decrease the average Nusselt number whereas heat absorption increases it. Dawood et al. [10] investigated hydro-magnetic mixed convection double diffusive in a Lid Driven Square Cavity. Hussein [11] investigated the mixed convection in Square Lid-driven with Eccentric Circular Body. Munshi et al. [12] analyzed a numerical study of Mixed Convection in Square Lid-driven with internal elliptic body and constant flux heat source on the bottom wall. Nasrin [13] carried out aspect ratio effect of vertical liddriven chamber having a centered conducting solid on mixed magneto convection. Billah et al. [14] investigated the numerical analysis of fluid flow due to mixed convection in a lid-driven cavity having an heated circular hollow cylinder. Md Rahman et al. [15] studied unsteady mixed convection in a porous media filled liddriven cavity heated by semi-circular heaters.

The main aim of this work is to examine the effect of hydromagnetic mixed convection double lid-driven square cavity with inside elliptic heated block. Results will be presented by streamlines, isotherms, Nusselt number, and velocity profiles. 


\section{Physical Configuration}

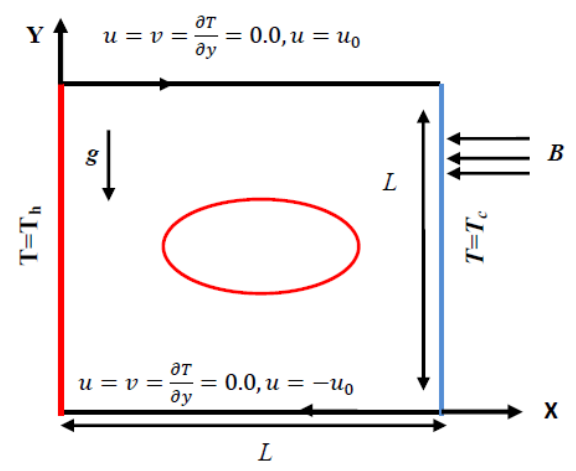

Fig. 1. Schematic diagram for the problem with boundary conditions upper surface move to right and lower surface move to left.

\section{Mathematical Formulation}

The governing equations describing the problem under consideration are based on the laws of mass, linear momentum and energy with buoyancy forces. The energy equation is written using the Boussinesq approximation. This means that all thermophysical properties of the fluid at a reference temperature are taken constant except in the buoyancy term of the momentum equation. In addition, the radition heat exchange is negligible in this study. The non-dimensional governing equations can be written as follows.

The equations are non-dimensionalized by using the following dimensionless quantities

$X=\frac{x}{L}, Y=\frac{y}{L}, U=\frac{u}{U_{0}}, V=\frac{v}{U_{0}}, P=\frac{p}{\rho U_{0}^{2}}, \theta=\frac{T-T_{c}}{T_{h}-T_{c}}$

where $v=\mu / \rho$, is the reference kinematic viscosity and $\theta$ is the non-dimensional temperature. After substitution of dimensionless variable we get the non- dimensional governing equations are:

$$
\begin{aligned}
& \frac{\partial U}{\partial X}+\frac{\partial V}{\partial Y}=0 \\
& U \frac{\partial U}{\partial X}+V \frac{\partial U}{\partial Y}=-\frac{\partial P}{\partial X}+\frac{1}{\operatorname{Re}}\left(\frac{\partial^{2} U}{\partial X^{2}}+\frac{\partial^{2} U}{\partial Y^{2}}\right) \\
& U \frac{\partial V}{\partial X}+V \frac{\partial V}{\partial Y}=-\frac{\partial P}{\partial Y}+\frac{1}{\operatorname{Re}}\left(\frac{\partial^{2} V}{\partial X^{2}}+\frac{\partial^{2} V}{\partial Y^{2}}\right)+R i \theta
\end{aligned}
$$


$U \frac{\partial \theta}{\partial X}+V \frac{\partial \theta}{\partial Y}=\frac{1}{\operatorname{Re} \operatorname{Pr}}\left(\frac{\partial^{2} \theta}{\partial X^{2}}+\frac{\partial^{2} \theta}{\partial Y^{2}}\right)$

Where, $\operatorname{Pr}$ is the Prandtl number, $R e$ is the Reynolds number, $R i$ is the Richardson number and $\mathrm{Ha}$ is the Hartmann number are defined as

$$
R e=\frac{u_{0}}{v}, R i=\frac{g \beta\left(T-T_{e}\right)}{u_{0}{ }^{2}}, P_{r}=\frac{\gamma}{\alpha} \text { and } H a=B L \sqrt{\frac{\sigma}{\mu}}
$$

The Dimensionless boundary conditions for left $\&$ right walls are:

$U=V=0, \theta=1$, at $X=0$

$U=V=0, \theta=0$, at $X=1$

The lower \& upper boundaries are:

At $Y=0, U=V=\frac{\partial \theta}{\partial Y}=0, U=-1$ (lower wall lid)

At $Y=1, V=\frac{\partial \theta}{\partial Y}=0, U=+1$ (upper wall lid)

\subsection{Nusselt Number calculation}

Equating the heat transfer by convection to the heat transfer by conduction at hot wall: $h \Delta T=-k\left(\frac{\partial T}{\partial x}\right)_{x=0}$

Introducing the dimensionless variables, defined in equation (1), into equation (6), gives:

$N u_{1}=-\left(\frac{\partial \theta}{\partial X}\right)_{X=0}$

The average Nusselt number is obtained by integrating the above local Nusselt number over the vertical hot wall:

$N u=-\frac{1}{A} \int_{0}^{A}\left(\frac{\partial \theta}{\partial X}\right)_{X=0} d Y$

\section{Numerical Technique}

The coupled governing partial differential equations, i.e., mass, momentum and energy equations are transferred into a system of integral equations by using the Galerkin weighted residual finite-element method. The integration involved in each term of these equations is performed with the aid Gauss quadrature method. The nonlinear algebraic equations so obtained are modified by imposition of boundary conditions. These modified nonlinear equations are transferred into linear algebraic equations with the aid of Newton's method. Lastly, Triangular factorization method is applied for solving those linear equations. For numerical computation and post-processing, the software COMSOL Multiphyics is used.

\subsection{Program validation and comparison with previous work}

The present numerical code is valided against the problem by Al-Amiri et al. [4] was modified and used for the computations in the study. The working fluid is chosen 
Prandtl number $\operatorname{Pr}=0.733$. The left wall is kept heated at $T_{h}$ and right wall is kept at cold $T_{c}$. The upper surface move to right and lower surface move to left. Streamlines and isotherms plotted in Fig. 2 are showing good agreement.

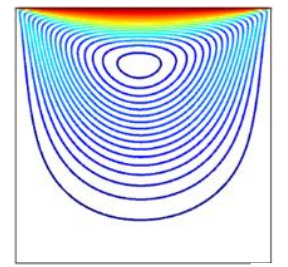

(a)

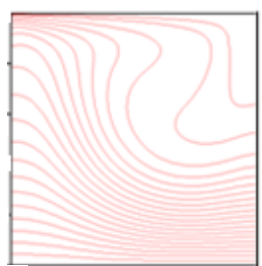

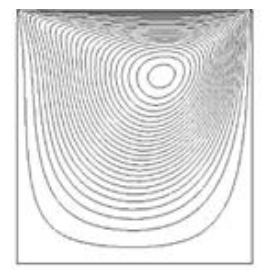

(b)

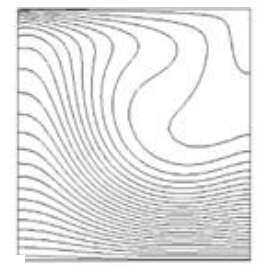

Fig. 2. Stream lines, isotherms for, $\operatorname{Pr}=0.733$ and $\mathrm{Re}=50$ (a) present code (b) Al-Amiri et al. [4].

\section{Results and Discussion}

The finite element simulation is performed to examine the laminar mixed convection flow and heat transfer in a square cavity having a centered elliptic cylinder. The mixed convection phenomenon in the cavity is influenced by Richardson number $R i$ ranging from $10^{3}$ to $10^{6}$, Reynolds number $R e$ varing from 50 to 150 and Prandtl number $P r=$ 0.733 respectively. Numerical computations are carried out and a parametric study is performed to illustrate the influence of the physical parameters on the resulting streamlines and isotherms as well as the velocity components at the enclose midsection, Nusselt number, velocity and temperature along the heat sources.

Streamlines for $R e=50$ and $P r=0.733$ are presented in Fig. 3 to understand the effect of Richardson number, $R i$ on flow field and temperature distribution. Most of the heat transfer occurs due to conduction except near the sliding right wall. Elliptic shape eyes formed at bottom wall of the cavity. For higher Richardson number, $R i$ elliptic shaped eyes are formed in the right wall of the cavity and also flow strength increases which are shown Fig. 3.

Conduction dominant heat transfer is obtained from the isotherms in Fig. 4 at $R i=$ $10^{3}$ to $R i=10^{6}$. With increases in Richardson number, $R i$, isotherms concentrates near the right wall so isotherm lines are more bending which means increasing heat transfer through convection.

Variation of Local Nusselt number, velocity, temperature and average Nusselt number with $\operatorname{Pr}=0.733$ and $R e=50$ inside the various elliptic diameter are shown in Fig. 5. When Richardson number $R i$ is small the local Nusselt number flow line near the bottom wall and Richardson number $R i$ increasing flow line increasing near the right wall. Fig. 5 shows the variation of the temperature and velocity profiles along the mid-sections of the cavity and the temperature distribution in most of the cavity is shown to retain similar behavior to that of a pure conduction regime except near the sliding top wall. In addition, Fig. 5 illustrates that the velocity components $U$ and $V$ 
exhibits higher values along their respective walls for higher values of Richardson number, $R i$.
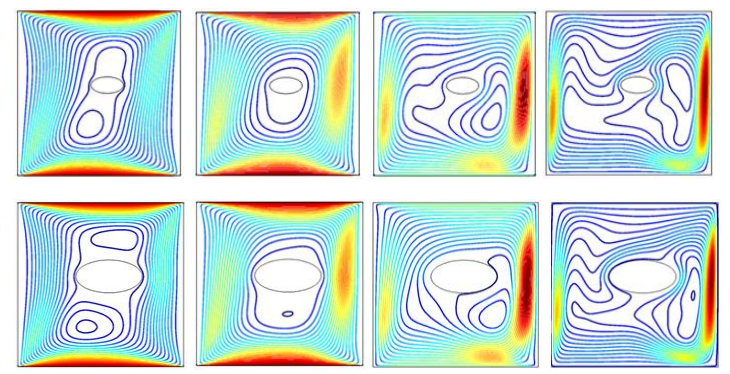

Fig. 3. Streamlines for $\mathrm{Gr}=10^{3}, 10^{4}, 10^{5}, 10^{6}$ when $\mathrm{Pr}=0.733$ and $\mathrm{Re}=50$ inside the various elliptic diameter.
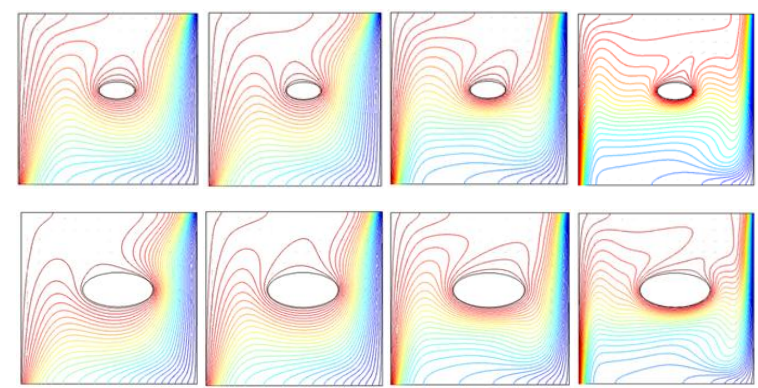

Fig. 4. Isotherms for $\mathrm{Gr}=10^{3}, 10^{4}, 10^{5}, 10^{6}$ when $\mathrm{Pr}=0.733$ and $\mathrm{Re}=50$ inside the various elliptic diameter.
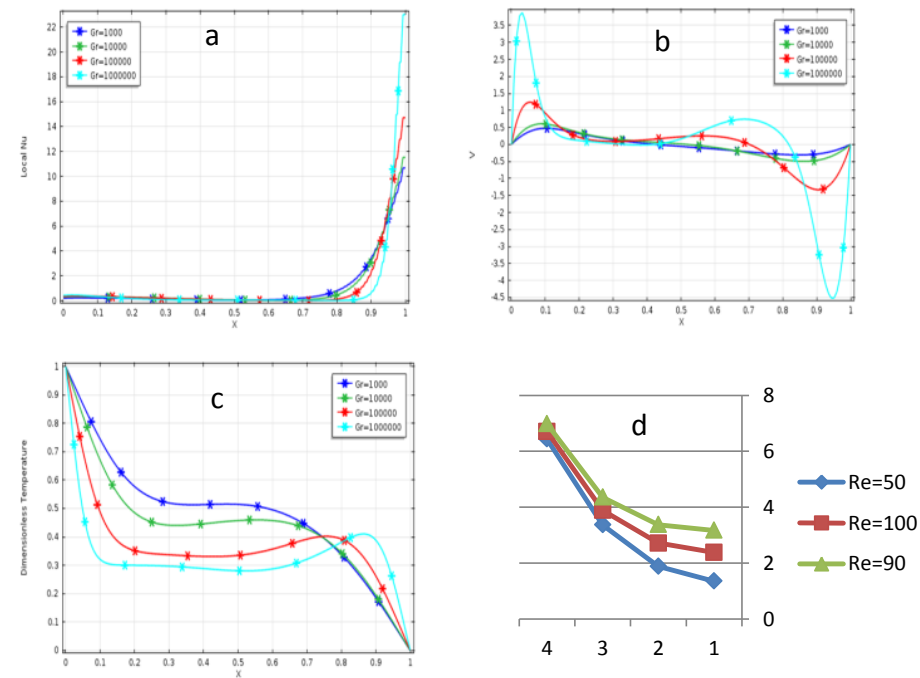

Fig. 5. Variation of (a) local Nusselt number, (b) velocity, (c) temperature and (d) average Nusselt number with $\operatorname{Pr}=0.733$ and $\operatorname{Re}=50$ inside the various elliptic diameter. 
Table 1. Average Nusselt Number Table for various Grashof number and Reynolds number.

\begin{tabular}{llll}
\hline $\mathrm{Re}$ & $\mathrm{Gr}$ & $\mathrm{Nu}$ \\
\hline & $10^{3}$ & 1.35318 & \\
50 & $10^{4}$ & 1.88436 & \\
& $10^{5}$ & 3.36277 & \\
& $10^{6}$ & 6.43441 & \\
& $10^{3}$ & 2.38517 & \\
& $10^{4}$ & 3.81826 & \\
& $10^{5}$ & 6.70534 \\
& $10^{6}$ & 3.16861 \\
& $10^{3}$ & 3.37567 & \\
& $10^{4}$ & 4.35792 & \\
& $10^{5}$ & 6.97807 & \\
\hline
\end{tabular}
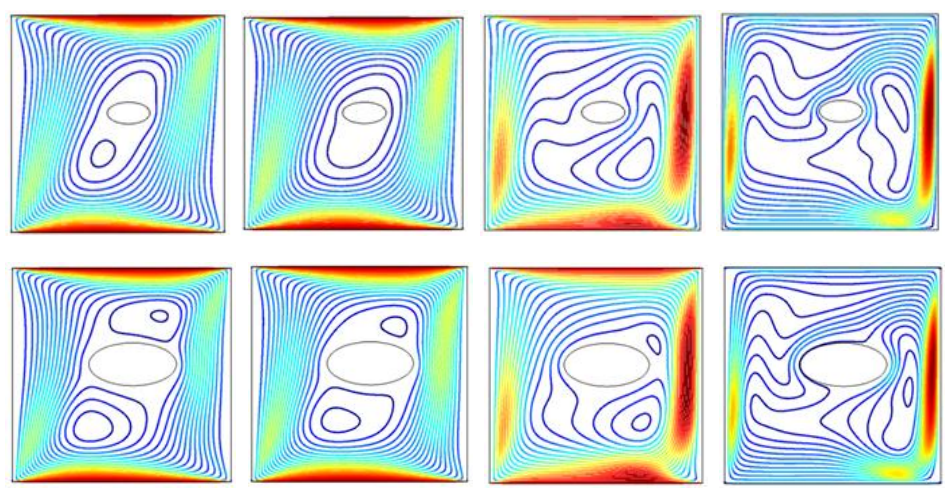

Fig. 6. Streamlines for $\mathrm{Gr}=10^{3}, 10^{4}, 10^{5}, 10^{6}$ when $\operatorname{Pr}=0.733$ and $\mathrm{Re}=100$ inside the various elliptic diameter.
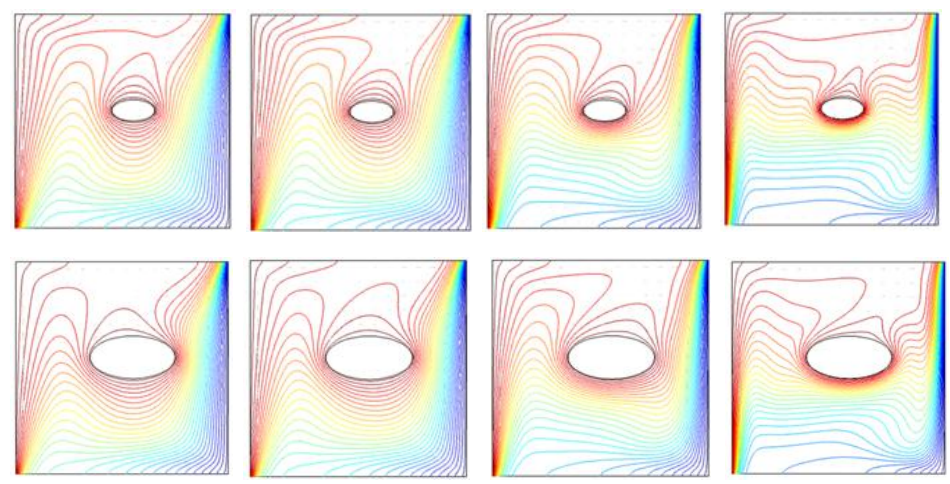

Fig. 7. Isotherms for $\mathrm{Gr}=10^{3}, 10^{4}, 10^{5}, 10^{6}$ when $\mathrm{Pr}=0.733$ and $\mathrm{Re}=100$ inside the various elliptic diameter. 

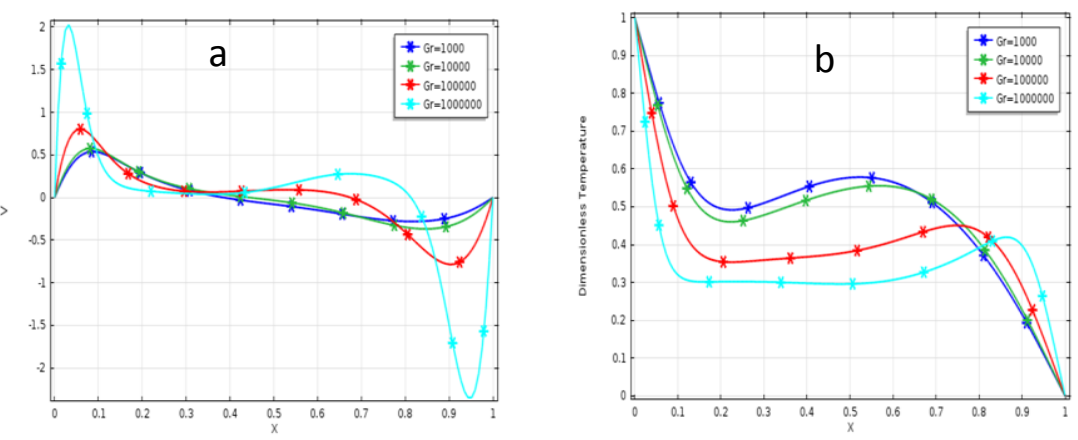

Fig. 8. Variation of (a) Velocity and (b) Temperature $\operatorname{Pr}=0.733$ and $\operatorname{Re}=100$ inside the various elliptic diameter.
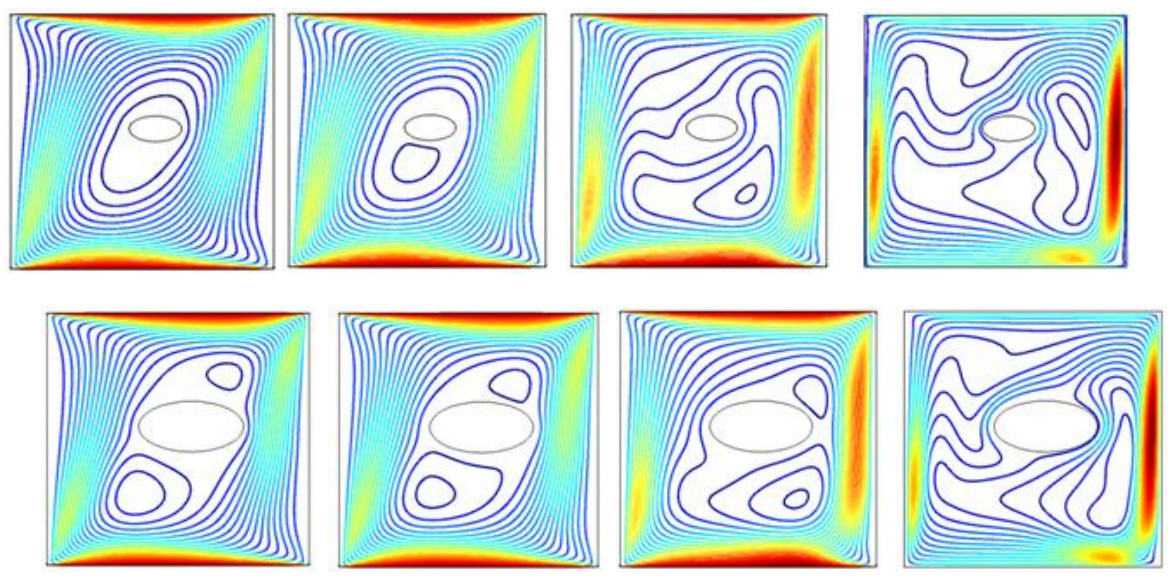

Fig. 9. Streamlines for $\mathrm{Gr}=10^{3}, 10^{4}, 10^{5}, 10^{6}$ when $\operatorname{Pr}=0.733$ and $\mathrm{Re}=150$ inside the various elliptic diameter.
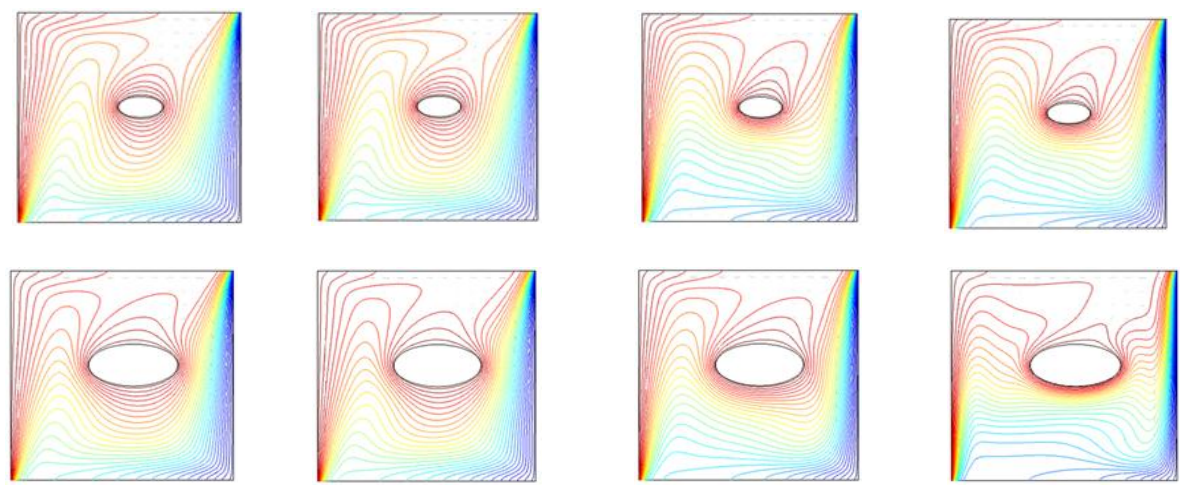

Fig. 10. Effect of Grashof number $\mathrm{Gr}=10^{3}, 10^{4}, 10^{5}, 10^{6}$ when $\mathrm{Pr}=0.733$ and $\operatorname{Re}=150$ (Isotherms) inside the various elliptic diameter. 

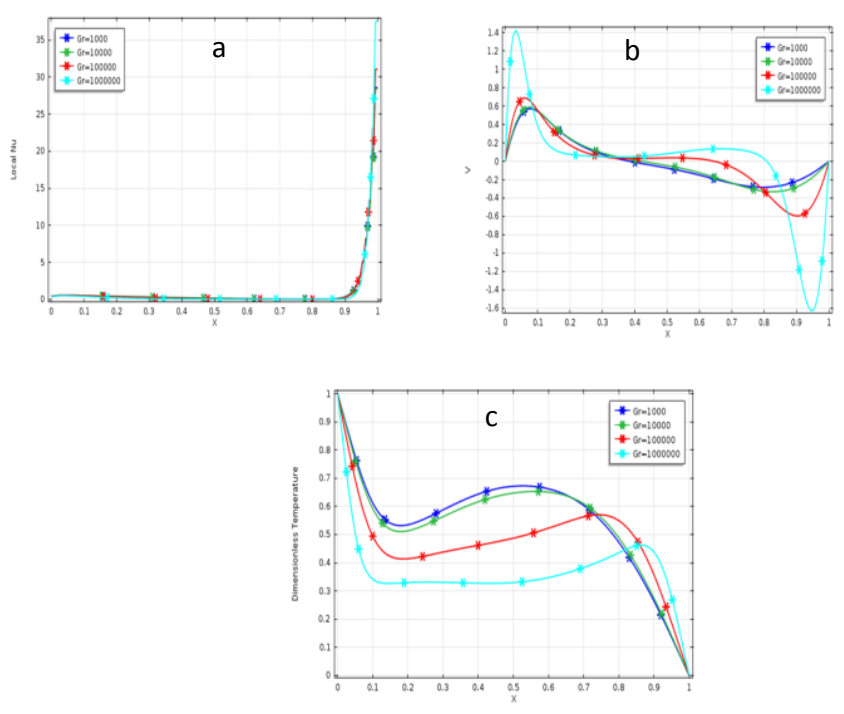

Fig. 11. Effect of Reynolds number on (a) Local Nusselt number, (b) Velocity, and (c) Temperature $\operatorname{Pr}=0.733$ and $\operatorname{Re}=150$ various elliptic diameters.

Variation of streamlines inside the cavity with Richardson number and Reynolds number are shown in Figs. 6 and 9, respectively. As can be observed from the figures with existence of the symmetrical boundary conditions about the vertical centerline of the cavity, the flow and temperature fields are symmetrical about this line. As can be seen from the streamlines for in the Fig. 6 and 9, a pair of counter-rotating eddies are formed in the left and right half of the cavity for all Grashof number and Reynolds numbers considered. Most of the heat transfer occurs due to conduction except near the sliding top and bottom wall. Elliptic shape eyes formed at bottom wall of the cavity. For higher Richardson number elliptic shaped eyes are formed in the right wall of the cavity and also flow strength increases which are shown figure.

Conduction dominant heat transfer is obtained from the isotherms in Figs. 7 and 10 at $R i=10^{3}$ to $10^{6}$. With increase in Richardson number $R i$, isotherms concentrate near the right wall so isotherm lines are more bending which means increasing heat transfer through convection.

Variation of local Nusselt number, velocity, temperature and average Nusselt number with $\operatorname{Pr}=0.733$ and $R e=100,150$ inside the various elliptic diameter are shown in Figs. 8 and 11. When Richardson number $R i$ small, the local Nusselt number flow line near the bottom wall and when Richardson number $R i$ increasing flow line increasing near the right wall. Figs. 8 and 11 shows the variation of the temperature and velocity profiles along the mid-sections of the cavity and the temperature distribution in most of the cavity is shown to retain similar behavior to that of a pure conduction regime except near the sliding top wall. In addition, Figs. 8 and 11 
illustrate that the velocity components $U$ and $V$ exhibits higher values along their respective walls for higher values $R i$.

\section{Conclusion}

Effect of hydro-magnetic mixed convection in a double lid driven square cavity with inside elliptic heated block has been performed. Results have been presented in terms of streamlines, isotherms, average Nusselt number at the heated block average temperature of the fluid and the temperature at the elliptic cylinder to analyze the effect of Grashof number, $G r$ and Reynolds number, $R e$ on the fluid flow and heat transfer in the cavity for the aforementioned Prandtl number, Pr. In view of obtained results, the following findings have been summarized:

a. Grashof number, $G r$ has a great significant effect on the streamlines and isotherms at the three convective regimes, Buoyancy- induced vorex in the streamlines increased and thermal layer near the heated surface become thin and concentrated with increasing Reynolds number, $R e$. The average Nusselt numbers at the heated surface is always upper and the average temperature in the cavity is inferior for the largest value of Reynolds number, $R e$.

b. Recirculation cell in the streamline plot decreases and thermal boundary layer thickness near the heated wall decreases strongly with increasing the values of Reynolds number, $R e$ at the three convective regimes. Maximum average Nusselt number, average fluid temperature and elliptic cylinder temperature are obtained, without the effect of Grashof number, $G r$, for the $P r=0.733$.

\section{Appendix: Nomenclature}

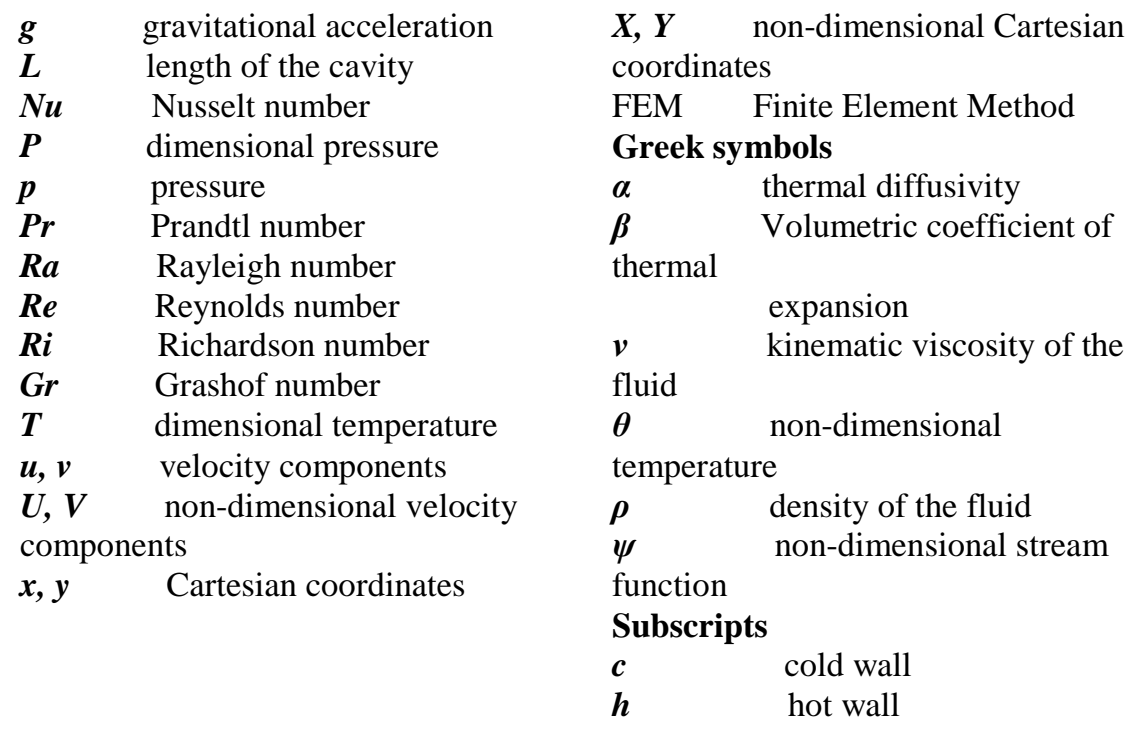




\section{Acknowledgments}

The author would like to express their gratitude to the Department of Mathematics, Bangladesh University of Engineering and Technology, for providing computer facility during this work.

\section{References}

1. L. A. B. Pilkington, Proc. R. Soc. A-Math. Phys. Eng. Sci. 314, 1 (1969).

2. C. K. Cha and Y. Jaluria, Int. J. Heat Mass Transfer 27, 1801 (1984). http://dx.doi.org/10.1016/0017-9310(84)90162-5

3. F. J. K. Ideriah, J. Mech. Eng. Sci. 22, 287 (1980). http://dx.doi.org/10.1243/JMES_JOUR_1980_022_054_02

4. A. M. Al-Amiri, K. M. Khanafer, and I. Pop, Int. J. Therm. Sci. 46, 662 (2007). http://dx.doi.org/10.1016/j.ijthermalsci.2006.10.003

5. M. A. R. Sharif, Appl. Therm. Eng. 27, 1036 (2007). http://dx.doi.org/10.1016/j.applthermaleng.2006.07.035

6. C. L. Chen and C.H. Cheng, Appl. Therm. Eng. 29, 2855 (2009). http://dx.doi.org/10.1016/j.applthermaleng.2009.02.009

7. K. M. Khanafer, A. M. Al-Amiri, and I. Pop, Eur. J. Mech. B/Fluids 26, 669 (2007). http://dx.doi.org/10.1016/j.euromechflu.2006.06.006

8. M. A. Teamah and W. M. El-Maghlany, Int. J. Therm. Sci. 49, 1625 (2010). http://dx.doi.org/10.1016/j.ijthermalsci.2010.04.023

9. L. K. Saha, K. M. Salah Uddin, and M. A. Taher, Am. J. Appl. Math. 3(1), 20 (2015).

10. M. M. K. Dawood and M. A. Teamah, Eur. J. Scientific Res. 85(3), 336 (2012).

11. N. A. Hussein, J. Babylon Univ./Eng. Sci. 21(2), 616 (2013).

12. M. J. H. Munshi, M. A. Alim, and G. Mostafa, Int. J. Eng. Appl. Sci. 3(6), 42 (2016).

13. R. Nasrin, J. Sci. Res. 3(3), 501 (2011). http://dx.doi.org/10.3329/jsr.v3i3.7433

14. M. M. Billah, M. M. Rahman, U. M. Sharif, N. A. Rahim, R. Saidur, and M. Hasanuzzaman, Int. Commun. Heat Mass Transfer, 38, 1093 (2011). http://dx.doi.org/10.1016/i.icheatmasstransfer.2011.05.018

15. M. M. Rahman, H. F. Oztop, S. Rahman, S. Mekhilef, and K. Al-Salem, Thermal Sci. 19(5), 1761 (2015). http://dx.doi.org/10.2298/TSCI130725098R 